\title{
A Comprehensive Analysis of MRI Research Risks: In Support of Full Disclosure
}

\author{
Jennifer Marshall, Toby Martin, Jocelyn Downie, Krisztina Malisza
}

\begin{abstract}
Magnetic resonance imaging (MRI) procedures have been used for over 20 years. This modality is considered relatively safe and holds great promise. Yet, MRI has a number of risks. In order for MRI research to meet the Canadian standard of disclosure, the investigator must communicate and make note of all risks in their research protocols and consent forms. Those creating and reviewing research protocols and consent forms must take notice of the different circumstances under which MRI poses a risk. First, this paper will describe the current standard of disclosure in Canada for research participants. Second, the paper will provide a comprehensive synthesis of the known physical and psychological risks associated with MRI. Third, the paper will provide recommendations concerning areas for further investigation and risk reduction strategies. This information will thus equip researchers and research ethics boards (REBs) with the criteria needed for the composition of research protocols that meet the Canadian disclosure standard.
\end{abstract}

RÉSUMÉ: Une analyse compréhensive des risques de la recherche en IRM, à l'appui de la divulgation complète. L'imagerie par résonance magnétique (IRM) est utilisée en clinique depuis plus de 20 ans. Elle est considérée relativement sûre et très prometteuse. Cependant l'IRM comporte certains risques. L'investigateur doit noter tous les risques dans ses protocoles de recherche et les indiquer dans les formulaires de consentement, afin que la recherche en IRM rencontre les standards canadiens sur la divulgation. Ceux qui élaborent les protocoles de recherche et les formulaires de consentement ainsi que ceux qui les révisent doivent signaler dans quelles conditions l'IMR comporte des risques. Cet article décrit d'abord les standards canadiens actuels de divulgation aux sujets de recherche, puis présente une synthèse extensive des risques physiques et psychologiques connus associés à l'IRM et enfin formule des recommandations, dans le corps de l'article et sous forme de table sommaire, concernant les aspects qui doivent être investigués davantage et les stratégies de réduction des risques. Cette information fournira aux chercheurs et aux comités d'éthique de la recherche les critères nécessaires à la rédaction de formulaires de consentement et de protocoles de recherche qui rencontrent les standards canadiens de divulgation.

Can. J. Neurol. Sci. 2007; 34: 11-17

Magnetic resonance imaging (MRI) is often championed in medical research circles because the modality is generally deemed safe and may be performed repeatedly. However, it is also clear that MRI research carries risks. As discussed in this paper, the legal and ethical standard for disclosure of risks in research requires that all known and foreseeable risks must be disclosed to the research participant or substituted decisionmaker. Given these two facts, one would expect that a clear, concise, and complete description of the risks of MRI would be available in the literature; however, this is not so. After a perusal of the literature, we could find only limited risk descriptions. While MRI risks have indeed been described in the literature, ${ }^{1-3}$ no article is comprehensive, detailing all risks. This detailed list and MRI safety information would be of great use to the neurological science community. This paper brings together

\footnotetext{
From the Health Law Institute, Dalhousie University (JM, JD), Halifax, NS; St. Amant Centre (TM), Winnipeg, MB; Institute for Biodiagnostics, National Research Council (KM), Winnipeg, MB, Canada.

ReCEIVED MAY 23, 2006. ACCEPTED IN FINAL FORM OCTOBER 28, 2006. Reprint requests to: Jennifer Marshall, Dalhousie University, Health Law Institute, 6061 University Avenue, Halifax, NS, B3H 4H9, Canada.
} 
information on the Canadian standard of disclosure of research risks and the risks of MRI research. Recommendations will also be made concerning areas in need of further investigation, risk reduction strategies and important criteria for the composition of consent forms and research protocols in order to meet the Canadian disclosure standard.

A literature review was undertaken in order to assemble a comprehensive overview of physical and psychological risks associated with MRI. Searches were carried out using PubMed, Ovid Online, and PsychINFO databases using search terms such as, "safety and MRI/magnetic field," "risk and MRI/magnetic field," "claustrophobia/anxiety and MRI," and others. In addition, searches were made using publicly available information from the Canadian Medical Association (CMA), the American Medical Association (AMA), the American Academy of Neurology (AAN), the American College Radiology (ACR), the US FDA, Health Canada, the American Society of Anesthesiologists (ASA), American Academy of Pediatrics (AAP), the Joint Commission on Accreditation of Healthcare Organizations (JCAHO) and MRI Safety websites.

This précis is intended to assist Research Ethics Boards (REBs) when reviewing protocols and consent forms and to educate more broadly on MRI research risks. To that end, the current legal and ethical standard for disclosure of risks for research involving humans in Canada will be explained. A synthesis of the physical and psychological risks associated with MRI research will then be provided. A sample template text of all MRI risks is available at www.neuroethics.ca for use by researchers and REBs.

This paper specifically focuses on risks of MRI research and the need for full disclosure of these risks. The issue of disclosure of risks in a clinical (as opposed to research) context, as well as issues surrounding the risks of fMRI (functional MRI), and other emerging neuroethics topics such as thought privacy or neuroimaging databases will not be examined in this paper.

\section{Current Standards}

\section{Disclosure}

In Canada, the legal standard of disclosure of risks for research with no intended benefit for participants has been addressed by two court decisions. In Halushka v. University of Saskatchewan, the Saskatchewan Court of Appeal established that the duty of disclosure of investigators to research participants is "at least as great as, if not greater than, the duty owed by the ordinary physician or surgeon to his patient" (1965), 53 D.L.R. (2d) 436 (Sask. C.A.). The court also found that the physician privilege of withholding information from the patient "so that he should not worry" has no application in the research context (Ibid., at 444). In Weiss v. Solomon, the Quebec Supreme Court established that all risks, even those rare and remote, must be disclosed to the research participant, especially if these risks could have serious consequences. The court in Weiss based its decision on the disclosure requirements found in Halushka as well as the Declaration of Helsinki and the Civil Code of Quebec. ${ }^{4}$

Beyond the courts, the Tri-Council Policy Statement on Research Involving Humans (TCPS) upholds that research participants must be provided with "a comprehensible description of reasonably foreseeable harms and benefits that may arise from research participation" (Art. 2.4c). ${ }^{5}$ Moreover, the TCPS requires that information regarding the "potential for physical or psychological harm" is also described to research participants (Art. $2.4 \mathrm{c}$ ). ${ }^{5}$

In summary, the current standard for disclosure for research involving humans in Canada is that all known and foreseeable risks - even those rare and remote-must be disclosed to the research participant or their substitute decision-maker. This means that when an investigator prepares, or an REB reviews, MRI research protocols and consent forms, care must be taken to ensure that all known and foreseeable risks are disclosed. The following is a description of the risks of MRI research as well as risk reduction strategies.

\section{RISK SyNTHESIS}

In this paper, MRI research risks have been broadly divided into two categories: physical and psychological. The MRI risks may also be categorized by those risks that are directly related to the MRI system or are associated with secondary procedures (e.g., sedation, contrast injection). Further risks could also be associated with any additional examinations required to respond to unexpected findings.

\section{Physical Risks}

\section{A. MRI magnet}

Magnetic resonance imaging systems use a strong static magnetic field, a pulsed gradient magnetic field and radiofrequency $(\mathrm{RF})$ energy to obtain images of the body in selected planes. ${ }^{6}$ A potentially problematic component of the MR environment is the strong static magnetic field (up to 100000 times the magnetic field strength of the earth) that is always present even when the scanner is not imaging. ${ }^{7}$ The pulsed gradient magnetic field used for signal localization and the RF coils used to elicit MR signals from tissue may also have potential risks. Some risks associated with the MRI magnet are known while others are unknown and in need of further investigation.

\section{KNOWN RISKS}

\section{Static Magnetic Field}

Metal objects (e.g., oxygen bottles, IV poles, pens, hair barrettes) can be strongly attracted to the MRI magnet (the "missile effect") and could cause serious injury to anyone in the MRI environment. For example, a hair barrette near a 1.5 T MRI magnet can reach speeds of $40 \mathrm{mph}$ (about $60 \mathrm{~km} / \mathrm{h}$ ). ${ }^{7}$ The ACR published its White Paper on MR Safety after an oxygen tank struck and killed a young boy during an MRI exam. ${ }^{8}$

The magnet may also cause metallic implants inside the body to move or electrically conductive material to heat up potentially causing severe burns. Therefore, implants such as, aneurysm clips are contraindications to MRI and scanning should not be performed where they are present. $^{7}$ A detailed list of MRI compatible surgical materials has been compiled to help determine patient and research participant safety during MRI examinations. ${ }^{9}$ 
An increasing number of people have implantable electronic or magnetic devices (i.e. pacemakers, defibrillators, cochlear implants) that can be disrupted by an MRI magnet, especially at the powerful field strengths that are presently in use. In some cases, medical devices are therefore contraindications to MRI and scanning should not be performed., ${ }^{7,9}$

The risks associated with metal in or around the magnet can be reduced through such strategies as screening questionnaires, preparatory CT scans, thermally insulating electrically conducive material and the use of MR compatible devices. ${ }^{1}$

Human tissues have very low magnetic susceptibility and lack enough ferromagnetic material to lead to harm due to MR imaging. ${ }^{10}$ To date there have been well over 100 million MRI examinations performed, and no evidence has appeared to support a claim that exposure to the magnetic field is unsafe at regulatory levels. ${ }^{11}$ In 2003, the US FDA published new criteria for MRI devices limiting field strengths to 8 Tesla for adults, children and infants older than one month and $4 \mathrm{~T}$ for neonates and infants. ${ }^{12,13}$ This new standard for MRI scanners provides a field strength threshold beyond which FDA ethical approval would be needed. Current Canadian guidance on MRI safety limits is considered outdate. ${ }^{14}$ Thus, Canadian researchers and clinicians must rely on the FDA guidelines which are more complete and accessible. Updated Canadian guidelines need to be provided by the appropriate regulatory agency.

\section{Time-Varying RF Magnetic Field}

While many hypothetical mechanisms for bioeffects of the RF field have been proposed, the only accepted biological concern is heating. ${ }^{1,15-17}$ Sensations of warmth occur only rarely and are always temporary. ${ }^{14}$ The amount of RF energy deposited in the patient during an MRI exam depends on the static magnetic field strength, the amount of energy applied by the RF pulse (number of pulses, type(s) of pulse(s), including flip angle and waveform), anatomy imaged, patient size, and coil design. The magnetic induction, however, occurs at the surface where heat dissipates more readily into the environment. ${ }^{15,18}$ The MRI system fans typically provide sufficient cooling from the increased temperature caused by RF absorption. It is therefore important that the fan is running during high energy sequences.

The RF energy absorbed by the patient can be calculated in terms of Watts/kg and is known as the specific absorption rate (SAR). Specific absorption rate is limited by FDA and Health Canada regulations and depends on certain environmental conditions, such as temperature, humidity, air and blood flow rates and insulation. The typical human metabolic rate is approximately 1.3 Watts $/ \mathrm{kg}$, while highly trained athletes raise their metabolic rate to nearly 18 Watts $/ \mathrm{kg}$ during vigorous exercises. The SAR for MRI exams is well below this level of vigorous exercise. ${ }^{15,18}$

Danger of localized heating exists where the subject may be exposed to sources of higher electric fields, for example, if they are near transmit coils, or they contact the magnet bore (as can happen with larger patients). Padding can be used to insulate the subject from these effects and formations of closed loops, such as the subject clasping hands, should be avoided. Heating should be limited when using local transmit coils to prevent burns. ${ }^{18}$ Researchers and REBs should pay particular attention to potential heating problems when new coils are being tested.
At higher field strengths, MRI may also induce an electric field within the body that can cause peripheral nerve stimulation or tingling producing an unpleasant or even painful sensation. ${ }^{1}$ Considered a serious concern by the US FDA, patients have also reported seeing spots (magnetophosphenes) thought to result from a slight torque (movement created by magnetic material aligning with the MRI magnet) created by the pulsed gradient magnetic field exerted on the retinal cones. ${ }^{7}$ These effects disappear when exposure ceases and do not affect visual acuity, presenting no apparent health hazard. ${ }^{17}$ As with SAR, there are safety regulations limiting the rates of change in magnetic flux density produced by the gradient coils $(\mathrm{dB} / \mathrm{dt}){ }^{7,13}$

\section{Noise}

The loud acoustic noise that can be produced by the MRI scanner could cause hearing loss if appropriate sound protection is not used. ${ }^{3}$ The factors that can affect the amount of hearing damage include the duration of the sound, frequency, intensity and temporal pattern. Modern MRI systems have significantly reduced noise levels, however, appropriate hearing protection is still recommended. Furthermore, certain drugs are known to increase hearing sensitivity and so the risk of physical discomfort may be increased in some individuals. ${ }^{19}$ In addition, neonates with immature anatomic development may react strongly to the loud noise of the MRI scanner. ${ }^{20}$ Sedated patients also have an increased sensitivity to sudden and frequently monotonous MR noise. ${ }^{12}$

Ear plugs and/or headphones are typically employed to reduce this risk to hearing. For infants, soft ear putty and minimuff ear protection should be used. ${ }^{12}$ The risk can be reduced by using scanners that do not output the continuous maximum noise level associated with hearing loss.

\section{WORK IN NEED OF FURTHER RESEARCH}

\section{Magnetic field}

While MRI is considered to be relatively safe at higher magnetic field strengths (i.e., 8 Tesla for adults, 4 Tesla for neonates), an insufficient number of well-designed, controlled human studies have been conducted to justify claims of absolute safety. ${ }^{14}$ In the same way, long-term health risks from the magnetic field or radio frequency waves have not been proven. Furthermore, MR physics does not suggest that there would be any harmful effects of exposure to MRI within the approved guidelines. Nevertheless, long-term epidemiological studies have yet to be performed in order to unequivocally prove this. Although absolute safety may be difficult if not impossible to empirically demonstrate, ${ }^{1}$ short-term exposures have been extensively studied. Most of these studies conclude that there are no substantial harmful biologic effects to short-term exposure. ${ }^{1}$ Furthermore, the literature does not contain carefully controlled experiments demonstrating absolute safety with chronic exposure to strong magnetic fields. ${ }^{1,21}$ A commitment to disclose new findings about previously unknown risks should be made by the researcher to the REB and to research participants.

\section{Pregnancy}

To date, clinical MRI has produced no known deleterious effects during pregnancy. ${ }^{1}$ Nevertheless, the research community 
has generally refrained from imaging pregnant women unless the anticipated benefits are thought to outweigh the unknown risks. ${ }^{22}$ In any case, additional research is needed in order to determine whether MRI is safe during pregnancy. ${ }^{1}$ For example, a large scale retrospective chart review of those women who received a clinical MRI during pregnancy and children born of women who received a clinical MRI during pregnancy could be initiated.

\section{B. Secondary procedures}

Physical risks can arise from the MRI examination process that are not directly related to the MRI magnet. The administration of sedation or contrast enhancement, though not always required during the MRI exam, can carry potential serious risks for the research participant. In addition, unexpected findings can lead to additional unnecessary medical examinations that may carry potential physical (and psychological) risks. These risks, not directly related to the MRI magnet, are described below.

\section{Sedation}

The vast majority of individuals undergoing an MRI scan do not require sedation or anesthesia. However, under certain circumstances such as cognitive impairment, claustrophobia, severe pain or critical illness, sedation may be required. ${ }^{2}$ The risks of sedation (depending on the drug used, level of sedation, and age of participant, among other factors) commonly include drowsiness, confusion, impaired judgment, nausea, and vomiting. Rarely, complications may include difficulty breathing, brain damage, and death $(1: 250000) .^{23}$

After the MRI research scan, there may be residual risks while the research participant recovers from sedation. Children may be particularly vulnerable to a prolonged recovery from, and side effects of, sedation. ${ }^{24}$ These risks could be heightened when children have been released from care.

For safety while sedated, breathing, heart rate (ECG), blood oxygen level (pulse oximeters) and body temperature are monitored. Due to the powerful magnetic field and RF generation, the monitoring equipment may cause severe burns if appropriate precautions are not taken (i.e. MRI-compatible equipment, wire insulation, avoiding wire coiling). ${ }^{25}$ If the research participant has been sedated, the risk of serious injury may be greater. ${ }^{7}$

The risks associated with the use of sedation can be reduced through various strategies. For instance, there are welldeveloped sedation guidelines by the American Society of Anesthesiologists, ${ }^{26}$ American Academy of Pediatrics ${ }^{24,25}$ and the Joint Commission on Accreditation of Healthcare Organizations. ${ }^{27}$ Trained personnel can be assigned to monitor the research participant while they are sedated and until they have sufficiently recovered from sedation.

These risks and the use of risk reduction strategies should be explained in the protocol and consent form. Parents or guardians can be educated regarding the potential for delayed adverse events and the need for continued observation at home.

\section{Contrast enhancement}

Contrast agents, usually gadolinium-based mixtures, may be used to improve contrast between tissue types. The injection of contrast agents through an IV needle can cause discomfort, generalized coldness, a tingling sensation in the throat, nausea, hives or, headache. ${ }^{1}$ Although very rare, some allergic reactions to contrast agents may be life-threatening (about $0.0003 \%$ incidence). The total incidence of adverse events for MRI contrast agents is less than $5 \% .^{9}$ According to the ACR White Paper on MR Safety, the risks of MRI contrast agents are sufficiently high that they should not be routinely used in pregnant patients ${ }^{22}$ and, therefore, should never be used in pregnant research participants.

The risks associated with the use of contrast agents can be reduced through such strategies as ensuring the presence of healthcare workers and resuscitation equipment at the scene.

\section{Unnecessary additional examinations}

Magnetic resonance imaging research gives rise to the possible discovery of unexpected findings, such as an abnormality or disease. According to several articles on the discovery of unexpected findings, one to two percent of adult and paediatric research participants' MRI research examinations reveal medically significant findings (e.g. tumours, aneurysms). ${ }^{28,29}$ As a result of the detection of unexpected findings, a research participant may require invasive examinations with the attendant potential for related medical complications. Occasionally these examinations will lead to discovery of treatable conditions. Sometimes, however, these findings will reveal nothing and therefore the increased physical risks of the ultimately unnecessary additional examinations will have been carried out with no attendant benefit. The potential for physical risks under these circumstances needs to be disclosed.

\section{Psychological Risks}

Magnetic resonance imaging scans present psychological risks, though they are not intrinsic to the imaging technology. They are not, for example, the result of the magnetic field directly affecting the brain. Negative psychological effects are instead due to situational characteristics, probably including the closeness of the scanner bore, the loud noise it makes, the long duration of required immobility, and the patient's anxiety about what the test may reveal. ${ }^{30,31}$ Exacerbating or secondary circumstances may also influence levels of anxiety during a MRI exam. The MRI noise can result in verbal communication difficulties during the MRI exam and/or heightened anxiety or discomfort in individuals with psychiatric disorders or those individuals that need to be sedated, the elderly or paediatric patients. ${ }^{1}$ Individuals with psychiatric illnesses may be predisposed to suffer greater distress during MRI procedures. ${ }^{32}$ All of these stimuli may lead to both transitory and long-term negative effects.

\section{A. Transitory effects}

The MRI scan situation may produce fear or distress during the scan itself, and at times immediately preceding or following the scan. These reactions are variously described (though rarely defined) in research reports as fear, anxiety, panic, or claustrophobia. 


\section{Panic}

Patients receiving MRI scans sometimes experience panic, often resulting in scan termination. Panic was diagnosed in 46 of 3000 adults $(1.5 \%)$ undergoing an MRI exam, ${ }^{33}$ according to whether the attending physician observed at least four out of ten clinical symptoms (e.g. chest pain, vertigo, sweating, and nausea).

A larger proportion of self-reported panic was observed in a sample of 80 adults. ${ }^{34}$ Following their scans, subjects read a brief description of a panic episode, and then stated whether or not they had experienced panic during the scan. Eleven $(13.8 \%)$ subjects answered affirmatively; three of these had terminated the scan early.

\section{Self-reported anxiety}

Researchers have measured fear by delivering brief questionnaires that require patients to rate agreement with statements about their feelings, usually on a 4 or 5 point Likert scale. The "state" portion of the State-Trait Anxiety Inventory (STAI) has been used most frequently, and consists of 20 items with which respondents may agree not at all (1 point), somewhat ( 2 points), moderately so ( 3 points), or very much so (4 points). Final scores therefore range from 20 to 80 , and normal (mean) values for state anxiety in a healthy adult population are 38.1 $(\mathrm{SD}=10.1)$ for women, and $37.3(\mathrm{SD}=9.8)$ for men. ${ }^{35}$

Risk is described usefully by identifying the relative frequencies of various magnitudes of harm. We wish to know, for example, the proportions of patients who report mild, moderate, or severe distress levels. Unfortunately, characterizing risk has not been the focus of most studies that measured distress caused by MRI scans.

In two studies, post-scan STAI questionnaire items were reworded to require retrospective evaluation of anxiety experienced during the scans. One study found a mean state anxiety level of $37.1(\mathrm{SD}=11.6)$ among men, and $43.1(\mathrm{SD}=14.6)$ among women. ${ }^{36}$ The other study reported an overall mean state anxiety score of 38.54 (SD 13.0). ${ }^{37}$ In a number of other studies, ${ }^{31,38-41}$ state anxiety was measured both before and after MRI scans, but the post-scan questions requested current, not retrospective reports. Pre-scan anxiety sample means in these studies ranged from 35.5 to 47.31. Post-scan means ranged from 33.0 to 45.24 .

\section{B. Long-term effects}

The psychological effects of MRI scans may include learning, meaning that however a patient responds during an MRI scan, his or her future responses to the same or similar situations may conceivably be affected.

\section{Effects on future MRI reactions}

Fear responses to MRI scans could sensitize or habituate, meaning that a patient might report more or less fear, respectively, the next time that an MRI situation is experienced or contemplated. The best evidence for this effect would come from repeated fear measurements of the same participants across multiple MRI scans, but to date no such data are available. Fear measurements from a single scan can also indicate learned reactions if participants with previous scanning experience are compared to those without it. In one such study, ${ }^{40}$ pre-scan anxiety ratings were not significantly different between those who had previously had MRI experience and those who had not. Some evidence that patients habituate to the MRI experience, even if initially stressful, comes from the success of exposurebased treatments for MRI scan anxiety $42 .{ }^{42,43}$ These procedures involve repeatedly presenting the fear-eliciting stimuli and situations under controlled conditions, with the result (in both studies cited) that the situations come to elicit little or no fear.

\section{Claustrophobia}

Of great concern is the possibility that an MRI experience may increase fear responses to new (but probably related) stimuli and situations. Could MRI scans cause claustrophobia, in other words - a disorder characterized by the marked, persistent and excessive fear of enclosed spaces $?^{32}$ As with sensitization/ habituation, strong evidence would involve measurements of claustrophobic behaviour recorded in the problem situation(s) both before and after the MRI scan. For example, a patient may receive a scan, experience distress, and subsequently experience a panic attack while trapped briefly in a small elevator. But unless the patient had been similarly confined before the scan and had experienced much less anxiety, the claim that the scan was responsible for the panic attack would be weak.

At least one published report apparently describes this phenomenon. Two adults without history of claustrophobia but who had trouble tolerating MRI scans subsequently reported persistent claustrophobia requiring psychiatric treatment. ${ }^{44}$ The authors acknowledged that case reports should be regarded cautiously in the absence of experimental demonstrations, however, and urged additional research.

Fear and anxiety questionnaires (such as the STAI) delivered prior to MRI scans have in several subsequent studies been represented at one month or later follow-up assessment. Even this design permits only limited conclusions about the link between MRI scans and claustrophobia. Patients may indicate greater claustrophobia on follow-up questionnaires mainly because those questionnaire items now evoke recollection of a specific, recent, and relatively unpleasant experience, and not because their reactions to constrained environments in daily life have changed.

For example, participants responded to STAI, Claustrophobia Questionnaire (CLQ), and MRI-Fear Survey Schedule (MRIFSS) tests both shortly before and one month following an MRI scan. ${ }^{36}$ At the follow-up, participant anxiety showed a statistically significant decrease on the STAI, no significant change on the CLQ, and a significant anxiety increase on the MRI-FSS. The MRI-FSS is a recent modification to the Fear Survey Schedule with items designed to address specific features of MRI scanning environments that may elicit fear. Given that CLQ scores (which measure claustrophobic reactions more generally) did not change, these results may be stronger evidence of sensitization (i.e. of reaction only to MRI scans) than of induced generalized claustrophobia.

\section{Anxiety related to unexpected findings}

The discovery of an unexpected finding on a MRI research scan may cause the participant to suffer psychologically. The psychological risks may include anxiety due to the detection of 
an untreatable asymptomatic abnormality or anxiety while waiting for follow-up investigations. In addition, some findings, upon review by a radiologist, may prove to be trivial, resulting in unwarranted worry. Feasible risk reduction strategies are being investigated in this area. Ways of dealing with unexpected findings need to be fully developed in order to reduce anxiety for research participants and researchers.

\section{Other risks associated with unexpected findings}

The detection of unexpected findings can have an effect on the research participant's insurability or employability. ${ }^{45,46}$ In the paediatric participant, findings of symptomatic untreatable abnormalities could carry the risk of lifelong implications, such as, profiling, stigmatization, discrimination and diminished expectations and opportunities.

\section{CONCLUSION}

Magnetic resonance imaging is considered by the research and medical community to be safe compared to other more invasive neuroimaging modalities. However, MRI is not without risk. Research participants need to be made aware of all potential harms. Those involved with research ethics review ought to be confident that all potential harms are being disclosed in a detailed manner. The lack of comprehensive documentation of all MRI risks in the science and medical literature would suggest that this is an area in need of clarification. A comprehensive document listing risk and MRI safety information would be of great use to the neurological science community as it is closely involved in MRI research.

In Canada, all risks need to be disclosed to research participants or their substitute decision-makers. Research Ethics Boards should therefore demand and researchers should provide protocols and consent forms that accurately disclose (1) all of the known risks; (2) any gaps in knowledge regarding risks; and (3) any risk reduction steps being used. Template text for consent forms based on this paper that could be used by REBs and researchers is available at www.neuroethics.ca. Importantly, issues regarding psychological risks remain. In particular, more research is needed to provide a complete picture of the psychological risks of MRI scans, particularly regarding longterm effects. Self-reported fear data from future studies should be presented as frequency distributions, to provide research ethics boards and prospective participants with specific probabilities of experiencing various levels of fear. Perhaps most importantly, as yet we know little about how the psychological risks of MRI scans may differ between clinical and research settings.

In this way, evidence-based practice guidelines, research protocols and consent forms can be developed and research participants can be better protected through MRI research meeting Canadian legal and ethical disclosure standards.

\section{REFERENCES}

1. Shellock FG, Crues JV. MR procedures: biologic effects, safety, and patient care. Radiology. 2004;232:635-52.

2. Gooden CK. Anesthesia for magnetic resonance imaging. Curr Opin Anesthesiology. 2004;17:339-42.

3. Chung SM. Safety issues in magnetic resonance imaging. J NeuroOphthalmol. 2002;22:35-9.
4. Glass KC, Lemmens T. Research involving humans. 2nd ed. In: Downie J, Caulfield T, Flood C, editors. Canadian Health Law and Policy. Markham: Butterworths; 2002. p. 459-500.

5. Tri-Council Policy Statement (TCPS): Ethical conduct for research involving humans. Ottawa: Public Works and Government Services Canada; 2003.

6. Health Protection Branch, Environmental Health Directorate, Minister of National Health and Welfare. Safety Code-26: Guidelines on exposure to electromagnetic fields from magnetic resonance clinical systems. Ottawa (Canada);1987.

7. Center for Devices and Radiological Health (CDRH) Magnetic Resonance Working Group. A primer on medical device interactions with magnetic resonance imaging systems. Washington, D.C.: US FDA; 1997.

8. Center for Devices and Radiological Health (CDRH). MRI safety. Washington, D.C: US FDA; 2001.

9. Shellock FG, Reference Manual for magnetic resonance safety, implants, and devices. 2004 ed. Los Angeles: Biomedical Research Publishing Company; 2004.

10. Schenck JF. Physical interactions of static magnetic fields with living tissues. Prog Biophys Mol Biol. 2005;87:185-204.

11. Shellock FG, Crues JV. MR Safety and the American College of Radiology White Paper. AJR. 2002;178:1349-52.

12. Kean M. MR safety in the pediatric MR environment. SMRT Educational Seminars, vol. 8(3) p. 20-30.

13. US Food and Drug Administration (US FDA). Guidance for industry and FDA staff: criteria for significant risk investigations of magnetic resonance diagnostic devices. Washington D.C.; 2003.

14. Harding P. Guidelines for ethics approval of research protocols involving human exposure to magnetic resonance imaging. London (Canada): University of Western Ontario; 2000.

15. Schaefer DJ. Safety aspects of radiofrequency power deposition in magnetic resonance. Magn Reson Imaging Clinics $\mathrm{N}$ Am. 1998;6:775-89.

16. Kanal E. An overview of electromagnetic safety considerations associated with magnetic resonance imaging. Ann NY Acad Sci. 1992;649:204-24.

17. Athey TW. Current FDA guidance for MR patient exposure and considerations for the future. Ann NY Acad Sci. 1992;649: 242-57.

18. Schaefer DJ, Felmlee JP. Radio frequency safety in MRI examinations. SMRT Educational Seminars. vol. 8(3) p. 11-19.

19. Laurell GF. Combined effects of noise and Cisplatin: short- and long-term follow-up. Ann Otol Rhinol Laryngol. 1992;101: 969-76.

20. Philbin MK, Taber KH, Hayman LA. Preliminary report: changes in vital signs of term newborns during MR. AJNR. 1996;17: 1033-36.

21. Bernhardt JH. Non-ionizing radiation safety: radiofrequency radiation, electric and magnetic fields. Phys Med Biol. 1992;37:807-44.

22. ACR White paper on magnetic resonance (MR) safety. Combined papers of 2002 and 2004. Reston: American College of Radiology; 2004.

23. Mayo Clinic. Anesthesia: Options and Considerations. [updated 2005 Oct 7; cited 2006 June 17]. Available from: http://www.mayoclinic.com/health/anesthesia/SC00026/.

24. American Academy of Pediatrics (AAP) Committee on Drugs. Prolonged recovery and delayed side effects of sedation for diagnostic imaging studies in children. Pediatrics. 2000; 105: $42-6$.

25. American Academy of Pediatrics (AAP) Committee on Drugs. Guidelines for monitoring and management of pediatric patients during and after sedation for diagnostic and therapeutic procedures. Pediatrics. 1992; 89: 1110-15.

26. American Society of Anesthesiologists (ASA). Updated Practice Guidelines for Sedation and Analgesia by Non-anesthesiologists. Park Ridge: American Society of Anesthesiologists (ASA); August, 2001. 
27. Joint Commission on Accreditation of Healthcare Organizations (JCAHO). Standards and intents for sedation and anesthesia care. Comprehensive Accreditation Manual for Hospitals. Chicago: JCAHO; 2002. Report No. TX 2-2.

28. Mamourian A. Incidental findings on research functional MR images: Should we look? ANJR. 2004;25:520-2.

29. Kim BS, Illes J, Kaplan RT, Reiss A, Atlas SW. Incidental findings on pediatric MR images of the brain. AJNR. 2002;23:1674-7.

30. Harris LM, Robinson J, Menzies RG. Evidence for fear of restriction and fear of suffocation as components of claustrophobia. Behav Res Ther. 1999;37:155-9.

31. Katz RC, Wilson L, Frazer N. Anxiety and its determinants in patients undergoing magnetic resonance imaging. J Behav Ther Exp Psychiatry. 1994;25:131-4.

32. Gollub RL, Shellock FG. Claustrophobia, anxiety, and emotional distress in the magnetic resonance environment. In: Shellock FG, editor. Magnetic resonance procedures: Health effects and safety. Boca Raton: CRC Press; 2001.

33. Avrahami E. Panic attacks during MR imaging: treatment with i.v. diazepam. AJNR Am J Neuroradiol. 1990;11:833-5.

34. McIsaac HK, Thordarson DS, Shafran R, Rachman S, Poole G. Claustrophobia and the magnetic resonance imaging procedure. J Behav Med. 1998;255-68.

35. Spielberger CD, Gorsuch RL, Lushene R, Vagg PR, Jacobs GA. Manual for the State-Trait Anxiety Inventory. Palo Alto: Consulting Psychologists Press; 1983.

36. Harris LM, Cumming SR, Menzies RG. Predicting anxiety in magnetic resonance imaging scans. Int J Behav Med. 2004; $11: 1-7$.
37. Lukins R, Davan IG, Drummond PD. A cognitive behavioural approach to preventing anxiety during magnetic resonance imaging. J Behav Ther Exp Psychiatry. 1997;28:97-104.

38. Dantendorfer K, Amering M, Bankier A, Helbich T, Prayer D, Youssefzadeh S, et al. A study of the effects of patient anxiety, perceptions and equipment on motion artifacts in magnetic resonance imaging. Magn Reson Imaging. 1997;15:301-6.

39. Kilborn LC, Labbé EE. Magnetic resonance imaging scanning procedures: development of phobic response during scan and at one-month follow-up. J Behav Med. 1990;13:391-401.

40. MacKenzie R, Sims C, Owens RG, Dixon AK. Patients' perceptions of magnetic resonance imaging. Clin Radiol. 1995;50:137-43.

41. Quirk ME, Letendre AJ, Ciottone RA, Lingley JF. Anxiety in patients undergoing MR imaging. Radiology. 1989;170:463-6.

42. Klonoff EA, Janata JW, Kaufman B. The use of systematic desensitization to overcome resistance to magnetic resonance imaging scanning. J Behav Ther Exp Psychiatry. 1986;17: 189-92.

43. Rosenberg DR, Sweeney JA, Gillen JS, Kim J, Varanelli MJ, O'Hearn KM, et al. Magnetic resonance imaging of children without sedation: preparation with stimulation. J Am Acad Psychoanal Dyn Psychiatry. 1997;36:853-9.

44. Fishbain DA, Goldberg M, Labbe E, Zacher D, Steele-Rosomoff R, Rosomoff $\mathrm{H}$. Long-term claustrophobia following magnetic resonance imaging. Am J Psychiatry. 1988;145:1038-9.

45. Grossman RI, Bernat JL. Incidental research imaging findings: Pandora's costly box. Neurology. 2004;62: 849-50.

46. Illes J, Rosen AC, Huang L, Goldstein RA, Raffin TA, Swan G, et al. Ethical consideration of incidental findings on adult brain MRI in research. Neurology. 2004;62: 888-90. 